\title{
Novel pharmaceutical molecules against emerging resistant gram-positive cocci
}

Authors

Roberto Manfredi, MD ${ }^{1}$ Sergio Sabbatani, $\mathrm{MD}^{1}$

${ }^{1}$ Department of Internal Medicine, Aging, and Nephrological Diseases, Division of Infectious Diseases, "Alma Mater Studiorum" University of Bologna, S. Orsola Hospital, Bologna, ItalyDiyarbakir, Turkey.
Submitted on: 03/18/2009 Approved on: 08/23/2009

Correspondence to: Roberto Manfredi, MD Associate Professor of Infectious Diseases, University of Bologna c/o Infectious Diseases, S. Orsola Hospital

Via Massarenti 11 I-40138 Bologna, Italy Tel: +39-051-6363355 Fax: +39-051-343500 E-mail: Roberto. manfredi@unibo.it

\begin{abstract}
Introduction: methicillin- and also vancomycin (glycopeptide)-resistant Gram-positive organisms have emerged as an increasingly problematic cause of hospital-acquired infections, also spreading into the community. Vancomycin (glycopeptide) resistance has emerged primarily among Enterococci, but the MIC values of vancomycin for the entire Staphylococcus species are also increasing worldwide. Material and Methods: the aim of our review is to evaluate the efficacy and tolerability of newer antibiotics with activity against methicillin-resistant and glycopeptide-resistant Gram-positive cocci, on the ground of our experience at a tertiary care metropolitan Hospital, and the most recent literature evidences in this field. Results: Quinupristin-dalfopristin, linezolid, daptomycin, and tigecycline show an excellent in vitro activity, comparable to the activity of vancomycin and teicoplanin for methicillin-resistant staphylococci, and superior to the one that vancomycin for vancomycin-resistant isolates. Dalbavancin, televancin, and oritavancin are new lipoglycopeptide agents with excellent activity against Gram-positive cocci, and have superior pharmacodynamics properties compared to vancomycin. We review the bacterial spectrum, clinical indications and practical use, pharmacologic properties, and expected adverse events and contraindications associated with each of these novel antimicrobial agents, compared with the present standard of care. Discussion: linezolid activity is substantially comparable to that of vancomycin in patients with methicillin-resistant Staphylococcus aureus (MRSA) pneumonia, although its penetration into the respiratory tract is exceptionally elevated. Tigecycline has activity against both Enterococus species and MRSA; it is also active against a broad spectrum of Enterobacteriaceae and anaerobes, which allows for use intraabdominal, diabetic foot and surgical infections. Daptomycin has a rapid bactericidal activity for Staphylococcus aureus and it is approved in severe complications, such as bacteremia and right-sided endocarditis. It cannot be used to treat pneumonia and respiratory diseases, due to its inactivation in the presence of pulmonary surfactant.
\end{abstract}

Keywords: resistant gram-positive cocci, staphylococci, enterococci, pneumococci, streptococci, epidemiology, clinical issues, novel antimicrobial compounds, characteristics, literature evidences.

[Braz J Infect Dis 2010;14(1):96-108] @Elsevier Editora Ltda.

INFECTIONS CAUSED BY GRAM-POSITIVE COCCI. AN OUTLINE OF THEIR ANTIBIOTIC RESISTANCE PROFILE

Gram-positive cocci have re-emerged as predominant pathogens of human hosts within the past 10-15 years. After the introduction of penicillin over 60 years ago, infections by Staphylococcus aureus, Streptococcus pyogenes, and Streptococcus pneumoniae finally became treatable. Within a short period of time, however, $S$. aureus developed resistance to penicillin. As a consequence, penicillinase-resistant penicillins were successfully introduced with in the early 1960's. Concomitantly, resistance emerged for the penicillinase-resistant penicillins, and finally methicillin (oxacillin)-resistant $S$. aureus
(MRSA) became a major hospital-acquired pathogen. Vancomycin (belonging to the class of glycopeptides) remained an active agent against MRSA and coagulase-negative Staphylococci, so that it was increasingly used during the subsequent years, until now. From the 1990's to the present, however, the emergence of resistance to vancomycin also occurred in a significant proportion. ${ }^{1-3}$ First among these organisms were Enterococcus faecium and Enterococcus faecalis. ${ }^{4}$ Subsequently, vancomycin (glycopeptide)-resistant enterococci (VRE) became a major hospital-acquired pathogen. In the past several years, MRSA were also spreading clonally into the community (the so-called "CA-MRSA"), leading to increased 
use of vancomycin-teicoplanin therapy. ${ }^{5}$ In the late 1990's, glycopeptide resistance was reported for coagulase-negative Staphylococci ${ }^{6}$ and then, S. aureus (the so-called vancomycin-intermediate S. aureus, or VISA, and the so-called glycopeptide-intermediate $S$. aureus, or GISA). The first reported isolation of VISA occurred in Japan in $1997^{7}$ and more than one hundred VISA isolates have been reported in the subsequent years. ${ }^{8}$ In the year 2002, three vancomycin-resistant $S$. aureus (VRSA) strains isolated from clinical specimens of American patients were found to have high level resistance to vancomycin (MIC $>32 \mathrm{ug} / \mathrm{mL}) .{ }^{9}$ Although a number of cases of VRSA have since been described, ${ }^{10}$ these isolates fortunately have not yet become widespread.

The re-emergence of Gram-positive cocci has been well established in the setting of hospital-acquired infections, but community-acquired infections due to MRSA have become increasingly problematic during the last years. ${ }^{11-13}$ Foreign body infections and bacteremia caused by coagulase-negative Staphylococci have also increased during time. ${ }^{14}$ As a result, vancomycin-teicoplanin usage has increased in both inpatients and outpatients. Although the majority of $S$. aureus strains remain susceptible in vitro to vancomycin, its efficacy against methicillin-sensitive S. aureus (MSSA) is inferior to that of penicillinase-resistant penicillins and beta-lactam derivatives as a whole. ${ }^{15,16}$

Actually, MRSA is born as a multi-drug resistant pathogen. Resistance to the macrolides, lincosamides, aminoglycosides, and all beta-lactam agents as a group, as well as fluoroquinolones, is also seen when MRSA is of clinical concern. Rifampin should not be used as a single agent due to rapid emergence of resistance in these microorganisms, while doxycycline and trimethoprim-sulfamethoxazole (cotrimoxazole) are bacteriostatic rather than bactericidal in their mechanisms of action. ${ }^{17}$

S. aureus is well known to be a virulent and invasive pathogen. It produces a variety of pyrogenic toxins and superantigens which contribute to its overall virulence. ${ }^{18}$ The presence of the Panton-Valentine leukocidin may predispose to invasive skin and soft tissue infections, and also necrotizing pneumonias and other necrotizing infectious localizations. MRSA infection often has its origin from a localized skin infection, with subsequent contiguous or hematogenous spread to lungs, heart (endocarditis), central nervous system (CNS), and sometimes bones and joints and other organs and sites. ${ }^{19}$ The prolonged duration of treatment with vancomycin or teicoplanin for severe infections, like endocarditis and osteomyelitis, may lead to more frequent and severe adverse effects (especially nephropathy, serum electrolyte imbalance, and myelotoxicity). While VISA/GISA and VRSA infections have only rarely been reported, clinical hetero-resistant populations of VISA (with strains showing MIC values $>4-16 \mathrm{mcg} / \mathrm{mL}$ ) have been isolated following prolonged administration of glycopeptides. Moreover, phar- macodynamics of vancomycin may have led to unappreciated under dosing of vancomycin, therefore predisposing to microbial resistance. ${ }^{20}$ Coagulase-negative Staphylococci have the capability to produce a glycocalyx enabling them to attach to prosthetic materials. ${ }^{21}$ Biofilm formation on the surfaces of medical devices (i.e. prosthetic devices, central vascular catheters), provides a protected environment for coagulase-negative Staphylococci; this biofilm formation impedes antibiotic penetration and reduces target site formation. ${ }^{21,22}$ As expected, catheter-related blood stream infections, CNS ventricular shunt infections, prosthetic joint infections, and prosthetic valve endocarditis are commonly caused by coagulase-negative Staphylococci. ${ }^{23}$

The large majority of these microorganisms usually are or become resistant to methicillin. Intermediate resistance to vancomycin was first reported among coagulase-negative Staphylococci several years before it occurred among S. aureus strains. Unlike S. aureus, infections by coagulase-negative Staphylococci on prosthetic hardware tend to be insidious and more chronic. Therapy often requires a combined medical- surgical approach with removal of the infected device and prolonged duration (usually exceeding four weeks) of antibiotic therapy thereafter.

Vancomycin-resistant Enterococci (VRE) are primarily associated with healthcare institutional acquisition in patients with co-morbid conditions. Since their peak incidence around the year 2000, several new antibiotics with excellent activity against VRE have been introduced into clinical practice in the meantime.

On the other hand, S. pneumoniae is the most frequent cause of community acquired pneumonia (CAP). It accounts for at least one third of patients with CAP. The crude incidence of this common pathogen rises to greater than $50 \%$, if respiratory culture with Gram stains and urinary antigen for S .pneumoniae are systematically performed. Associated bacteremia occurs in $20 \%$ of pneumococcal pneumonias and mortality is notably higher than for other respiratory pathogen. In vitro resistance of $S$. pneumoniae to penicillin as currently defined by Clinical Laboratory Standards Institute (CLSI) criteria, does not necessarily correlate with clinical failure. Specifically, penicillins have been favourably efficacious for pneumonia caused by penicillin-resistant pneumococci. ${ }^{24,25}$ These resistant isolates are often also resistant to macrolides, and in vitro resistance to macrolide does appear to correlate with clinical outcome. ${ }^{26,27}$

In adult patients, $S$. pneumoniae also represents the most common cause of meningitis. Empiric therapy for meningitis with ceftriaxone and vancomycin pending antibiotic susceptibility testing is often employed. Data from a large scale observational study of pneumococcal meningitis suggests that combination therapy may be superior to monotherapy ${ }^{28}$

Groups A Streptococci (whose leading organism is Streptococcus pyogenes), as well as other beta-hemolytic Streptococci, are often associated with life-threatening infections, especially involving the skin and soft tissues. Group B, C, F, and G beta- 
hemolytic Streptococci can also cause invasive infection and becteremia. Streptococcus agalactiae (belonging to group B Streptococci), is a common cause of neonatal sepsis. Fortunately, susceptibility to penicillin remains stable for the majority of the above-mentioned Streptococci.

\section{EPIDEMIOLOGICAL EXPERIENCE AT A MAJOR TERTIARY CARE HOSPITAL IN NORTHERN ITALY}

A prospective, microbiological surveillance study is ongoing for a decade at our academic Hospital (S. Orsola-Malpighi Hospital, Bologna, Italy), in order to check the epidemiological-clinical evolution of bacterial and fungal infections occurring among inpatients. All microorganisms isolated and identified from sterile sites (i.e. blood cultures, protected bronchoalveolar lavage, urine culture, and so on), are systematically tested for in vitro susceptibility against a consistent panel of antimicrobial compounds, and data are reported quarterly (A. Nanetti and S. Ambretti, unpublished data). Each bacterial isolate cultured from a single patient within one month is counted only once.
Some data regarding in vitro sensitivity rates of Staphylococcus aureus and Enterococci from the year 2005 to the first nine months of the year 2008 are reported in Table 1.

With regard to $S$. aureus, we underline a progressive reduction of methicillin resistance rate (from $56.8 \%$ of strains in the year 2005 up to $44.7 \%$ of overall tested strains in the year 2008), while we confirm a maintained $100 \%$ susceptibility to all available glycopeptides. Of major interest, the introduction of novel guidelines for a correct antimicrobial use in medicine and surgery seemed to lead to an apparently progressive reduction of the frequency of antibioticresistant strains, as demonstrated by an increased mean in vitro susceptibility rate against almost all tested compounds demonstrated in the year 2008, versus years 2005-2007, together with an apparent progressive trend toward reduced resistances during the examined temporal span (years 2005 to 2008). Furthermore, elevated sensitivity rates are also found for a number of "older" molecules, like cotrimoxazole $(87.9 \%$ to $98.2 \%)$, chloramphenicol $(83.5 \%$ to $88.6 \%)$, followed by rifampicin $(60.2 \%$ to $80.4 \%)$, clindamycin

Table 1. Microbiological figures from patients hospitalized at our tertiary-care hospital (S. Orsola-Malpighi Hospital, Bologna, Italy), year 2005-2008

\begin{tabular}{|c|c|c|c|c|}
\hline $\begin{array}{l}\text { Antibiotic susceptibility } \\
\text { (190 strains) }\end{array}$ & $\begin{array}{l}\text { Year } 2005 \\
\text { (167 strains) }\end{array}$ & $\begin{array}{l}\text { Year } 2006 \\
\text { (131 strains) }\end{array}$ & $\begin{array}{c}\text { Year } 2007 \\
\text { (Jan. to Sep.) } \\
\text { (103 strains) }\end{array}$ & Year 2008 \\
\hline Penicillin & 7.9 & 7.8 & 9.9 & 7.8 \\
\hline Amoxicillin-clavulanate & 43.2 & 48.5 & 48.9 & 55.3 \\
\hline Cefotaxime/Ceftriaxone & 42.9 & 48.5 & 48.9 & 55.3 \\
\hline Methicillin/Oxacillin & 43.2 & 48.5 & 49.6 & 55.3 \\
\hline Erithromycin & 34.2 & 48.5 & 51.9 & 55.3 \\
\hline Clindamycin & 34.9 & 48.5 & 51.9 & 54.4 \\
\hline Chloramphenicol & 84.7 & 88.6 & 87.8 & 83.5 \\
\hline Rifampicin & 60.2 & 64.0 & 62.1 & 80.4 \\
\hline Cotrimoxazole & 87.8 & 98.2 & 93.1 & 95.1 \\
\hline Gentamicin & 31.1 & 41.0 & 43.5 & 49.5 \\
\hline Vancomycin/Teicoplanin & 100.0 & 100.0 & 100.0 & 100.0 \\
\hline
\end{tabular}

In vitro antimicrobial susceptibility of Enterococci isolated from inpatients (years 2005-2008)

$\begin{array}{lcccc}\text { Antibiotic susceptibility } & \begin{array}{c}\text { Year 2005 } \\ \text { (206 strains) }\end{array} & \begin{array}{c}\text { Year 2006 } \\ \text { (151 strains) }\end{array} & \begin{array}{c}\text { Year 2007 } \\ \text { (155 strains) }\end{array} & \begin{array}{c}\text { Year 2008 } \\ \text { (Jan. to Sep.) } \\ \text { (108 strains) }\end{array} \\ \text { Penicillin } & & & & 50.9 \\ \text { Ampicillin } & 50.0 & 49.0 & 51.6 & 51.9 \\ \text { Tetracyclin } & 50.0 & 49.0 & 52.3 & 38.0 \\ \text { Vancomycin/Teicoplanin } & 46.6 & 45.0 & 38.7 & 96.3 \\ \text { Linezolid } & 98.1 & 95.4 & 94.8 & 100,0 \\ \text { Daptomycin } & 100,0 & 100,0 & 100,0 & 100.0 \\ \text { Quinupristin/Dalfopristin } & \mathrm{N} / \mathrm{A} & \mathrm{N} / \mathrm{A} & \mathrm{N} / \mathrm{A} & 100,0\end{array}$


(34.9\% to $54.4 \%)$, and erythromycin $(34.2 \%$ to $55.3 \%)$. These last compounds, which are largely available and do not imply increased costs of administration, are expected to be clinically effective alone and/or in combination with anti-Gram-positive agents (Table 1).

When examining the temporal trend of isolation of Enterococci (as a group) in the same time period (year 2005, up to September 2008), we notice a full sensitivity to novel compounds (i.e. linezolid, daptomycin, and quinupristin/dalfopristin), and a maintained in vitro effectiveness of glycopeptides ( $94.8 \%$ to 98.1\%). Vancomycin-resistant Enterococci (also called VRE) were isolated infrequently: only 9 cases in the year 2005, 6 in the year 2006, 8 in the year 2007, and only three cases in the first 9 months of the year 2009 (A. Nanetti and S. Ambretti, unpublished data). Also in the case of Enterococci, a number of "older" compounds still retain an effective activity against Enterococci, as demonstrated by susceptibility rates of penicillin (49.0\% to $51.6 \%$ ), ampicillin ( $49.0 \%$ to $52.3 \%$ ), and tetracyclines (38.0\% to $46.6 \%$ ). Also in this case, these compounds may act favourably or may be a part of a combination regimen, after in vitro sensitivity assays (Table 1 ).

\section{NOVEL ANTIBACTERIAL AGENTS WITH ENHANCED ACTIVITY AGAINST RESISTANT GRAM-POSITIVE COCCI}

The following antibacterial agents have been approved during the last five years: quinupristin/dalfopristin, ${ }^{29-32}$ linezolid, ${ }^{33,34}$ daptomycin, ${ }^{35-37}$ and tigecycline. ${ }^{38,39}$ Novel lipoglycopeptide agents under study include dalbavancin, ${ }^{40}$ telavancin, and oritavancin. ${ }^{41}$ Even novel cephalosporins (i.e. cefbiprole), and fluroquinolones (i.e. garenofloxacin) with enhanced activity against MRSA are in the pipeline. Some features of these novel antimicrobial molecule are summarized in Table 2 (microbiological spectrum, clinical indication, adverse events), and in Table 3 (selected pharmacological features).

\section{Quinupristin/Dalfopristin}

The so-called streptogramin antibiotic, quinupristin/dalfopristin, is a combination of two semisynthetic pristinamycin derivatives, which are represented by quinupristin and dalfopristin, in a 30:70 ratio. Resistance to quinupristin/ dalfopristin can occur by several mechanisms increasing enzymatic modification, active transport of specific efflux

Table 2. Novel antimicrobial agents for the management of resistant gram-positive infections. Microbiological, clinical, and therapeuticfeatures as of the end of 2008. [In vitro antimicrobial susceptibility of staphylococcus aureus strains isolated from inpatients (years 2005-2008)]

\begin{tabular}{|c|c|c|c|c|c|c|c|c|c|}
\hline \multirow[t]{2}{*}{ Drug } & \multirow{2}{*}{$\begin{array}{l}\text { Class } \\
\text { MRS } \\
\text { A }\end{array}$} & \multicolumn{4}{|c|}{ Microbiologically effective on } & \multirow[t]{2}{*}{ Adverse events } & \multicolumn{3}{|c|}{ Clinical indications } \\
\hline & & $\begin{array}{c}\text { MRS } \\
\mathrm{E}\end{array}$ & $\begin{array}{c}\text { PRS } \\
\mathrm{P}\end{array}$ & VRE & & & $\begin{array}{l}\text { Blood } \\
\text { stream }\end{array}$ & $\begin{array}{l}\text { Skin- } \\
\text { soft } \\
\text { tissue }\end{array}$ & $\begin{array}{l}\text { Hospital- } \\
\text { acquired } \\
\text { pneumonia }\end{array}$ \\
\hline Vancomycin & Glycopeptide & + & + & + & $\begin{array}{l}\text { Not VISA- } \\
\text { VRSA }\end{array}$ & $\begin{array}{l}\text { Nephro- and } \\
\text { ototoxicity } \\
\text { Red man } \\
\text { syndrome }\end{array}$ & + & + & + \\
\hline Teicoplanin & Glycopeptide & + & + & + & Not Van-A & $\mathrm{N} / \mathrm{D}$ & + & + & - \\
\hline $\begin{array}{l}\text { Quinupristin/ } \\
\text { Dalfopristin }\end{array}$ & Streptogramin & + & + & + & E. faecium & $\begin{array}{l}\text { Hepatic, phlebitis } \\
\text { Artho-myalgias }\end{array}$ & $\begin{array}{l}+ \\
+\end{array}$ & $\begin{array}{l}+ \\
+\end{array}$ & $\begin{array}{l}- \\
-\end{array}$ \\
\hline Linezolid & Oxazolidinone & + & + & + & + & $\begin{array}{l}\text { Neuropathy, } \\
\text { myelotixicity, } \\
\text { serotonin syndrome }\end{array}$ & + & + & + \\
\hline Daptomycin & Lipopeptide & + & + & + & + & $\begin{array}{l}\text { Arthro-myalgias, } \\
\text { CPK rise }\end{array}$ & + & + & - \\
\hline Tigecycline & Glycopeptide & + & + & + & + & Nausea, diarrhea & & + & - \\
\hline Dalbavancin & Glycopeptide & + & + & + & Not VAN-A & $\begin{array}{l}\text { Gastrointestinal, } \\
\text { hypokaleamia }\end{array}$ & + & + & - \\
\hline $\begin{array}{l}\text { Oritavancin } \\
-\quad-\end{array}$ & $\begin{array}{c}\text { Glycopeptide } \\
\text {-- }\end{array}$ & + & + & + & + & $\mathrm{N} / \mathrm{D}$ & - & + & - \\
\hline Telavancin & $\begin{array}{l}\text { Lipoglycopep- } \\
\text { tide }\end{array}$ & + & + & + & + & $\begin{array}{c}\text { Altered taste, CNS, } \\
\text { phlebitis }^{7}\end{array}$ & - & + & -- \\
\hline
\end{tabular}


Table 3. Novel Antimicrobial Agents for Management of Resistant Gram-positive Infections. Selected Pharmacogical Features Updated at the End of 2008

\begin{tabular}{|c|c|c|c|c|c|c|}
\hline \multirow[t]{2}{*}{ Drug } & \multirow[t]{2}{*}{ Class } & \multirow{2}{*}{$\begin{array}{c}\text { Pharmacodynamics } \\
\text { binding (\%) }\end{array}$} & \multirow{2}{*}{$\begin{array}{l}\text { Protein } \\
\text { route }\end{array}$} & \multirow{2}{*}{$\begin{array}{l}\text { Elimination } \\
\text { adjustment }\end{array}$} & \multicolumn{2}{|c|}{ Dosage } \\
\hline & & & & & Renal & Hepatic \\
\hline Vancomycin & Glycopeptide & AUC/MIC & $10-55$ & Renal & + & - \\
\hline Teicoplanin & Glycopeptide & AUC/MIC & 90 & Renal & + & - \\
\hline $\begin{array}{l}\text { Quinupristin/ } \\
\text { Dalfopristin }\end{array}$ & Streptogramin & AUC/MIC & N/A & Hepatic/feces & N/A & + \\
\hline Linezolid & Oxazolidinone & AUC/MIC & 31 & Hepatic & N/A & N/A \\
\hline Daptomycin & Lipopeptide & AUC/MIC & 92 & Renal & + & $\mathrm{N} / \mathrm{A}$ \\
\hline Tigecycline & Glycopeptide & Time above MIC & 68 & Biliary & N/A & + \\
\hline Dalbavancin & Glycopeptide & $\mathrm{AUC} / \mathrm{MIC}$ & $>95$ & Renal & + & $\mathrm{N} / \mathrm{A}$ \\
\hline Oritavancin & Glycopeptide & AUC/MIC & $\mathrm{N} / \mathrm{A}$ & Renal & Renal & $\mathrm{N} / \mathrm{A}$ \\
\hline Telavancin & Lipoglycopep- & AUC/MIC & $\mathrm{N} / \mathrm{A}$ & Renal & + & - \\
\hline
\end{tabular}

pumps mediated by an adenosine triphosphate-binding protein, and alteration of the target site. Resistance is rare for Streptococci and Enterococcus faecium. ${ }^{42}$ This streptogramin combination acts synergistically to inhibit bacterial protein synthesis at the ribosome level. Quinupristin/dalfopristin is therefore active against Staphylococcus aureus (including MRSA strains), Streptococcus pneumoniae, and Gram-positive anaerobes such as Clostridium spp., Peptococcus spp., and Peptostreptococcus spp. It is effective against vancomycin-sensitive as well as vancomycin-resistant Enterococcus faecium (VREF), but has little in vitro activity against Enterococcus faecalis, so it cannot be recommended until final speciation of Enterococcal organisms is concluded. Dalfopristin/quinipristin association inhibits cytochrome $\mathrm{P} 450$ $3 \mathrm{~A} 4$, and can inhibit agents metabolized through this pathway. Dosage adjustments may be needed in patients with hepatic dysfunction. Renal function has minimal impact on the agent's pharmacokinetics. A post-antibiotic effect is observed in 4-5 hours at 4X MIC for Staphylococci, 7-9 hours for Streptococci, and only 4 hours for Enterococci. ${ }^{43}$

The registered clinical indications for quinupristin/dalfopristin use include intra-abdominal infections, bacteremia, urinary tract infection and skin and soft tissue infections in which Enterococci may play a relevant pathogenic role. Overall clinical success rate for patients with vancomycinresistant E. faecium (VREF) proved to be $74 \%$, while overall clinical and bacteriological success rate was $66 \% .{ }^{44}$ Patients with bacteremia, those on mechanical ventilation, and those undergoing surgery had a worse outcome as might be expected. ${ }^{44}$ The most common and notable adverse events were arthralgias and myalgias, as well as vasculitis. In a comparative clinical trial of therapy for Gram-positive skin and soft tissue infections, $S$. aureus was the most frequent pathogen isolated. The clinical success rate of quinupristin/dalfopristin was comparable (68\%) to the comparator agents $(71 \%)$ (cefazolin, oxacillin or vancomycin). ${ }^{45} \mathrm{~A}$ higher incidence of drug-related adverse events occurred with quinopristin/dalfopristin as compared to other agents. ${ }^{46}$ For those patients receiving comparator agents, the most common reason for discontinuation was treatment failure (12\%). ${ }^{46}$ Furthermore, quinupristin/dalfopristin was compared to vancomycin in patients with hospital-acquired pneumonia. ${ }^{47}$ Successful outcomes were similar at $56 \%$ for quinupristin/dalfopristin and 58\% for vancomycin. The bacteriologic success rate was identical for both antibiotic groups, at around 54\% of treated cases. Quinupristin/dalfopristin has been also used to treat patients infected by $S$. aureus intolerant to or failing standard therapies. ${ }^{48}$

Ninety patients were treated for an average of 28 days with a $71 \%$ clinical outcome of cure or improvement and bacteriologic outcome of eradication or presumed eradication. Infections included bone and joint, skin and soft tissue, bacteremia, endocarditis, and respiratory tract involvement. Adverse events included mainly arthralgias (11\%), myalgias (9\%), and nausea (9\%). However, in patients with hepatic dysfunction or liver transplantation and concurrent receipt of immunosuppressive chemotherapy, the incidence of arthralgias approached $50 \%$ of treated subjects. ${ }^{49,50}$

\section{Linezolid}

Linezolid is an oxazolidinone antibiotic with activity against Gram-positive pathogens including VRE, MRSA, and VISA. The unique mechanism of action of linezolid involves the inhibition of bacterial protein synthesis through binding to the domain V regions of the 23 Sr RNA gene 46. Resistance to linezolid requires mutations of multiple gene copies, and seems an infrequent phenomenon. Linezolid is $100 \%$ bioavailable when given by either oral or intravenous route. Maximal plasma levels are achieved within 1-2 hours after oral dosing. Protein binding is only around 30\% with free 
distribution to well-perfused tissues. The drug does not require dosage alteration in the presence of renal failure, and no interaction exists for cytochrome P450 enzymes (and drugs metabolized through this last pathway). Linezolid and its two metabolites are decreased with hemodialysis, therefore dosing should occur post-dialysis. ${ }^{51}$

Linezolid is currently approved for skin and soft tissue infections and pneumonia due to susceptible pathogens. ${ }^{52}$ In two controlled trials of hospital-acquired pneumonia, a trend was seen for linezolid superiority over vancomycin. ${ }^{53,54}$ There is little data (mainly based on observational studies), on the utility of linezolid for either bacteremia, ${ }^{55}$ or osteomyelitis. ${ }^{56,57}$

Based on a rabbit model, linezolid does not have sufficient CSF penetration and should not be recommended for pneumococcal meningitis. ${ }^{33}$ However, CNS penetration appears adequate to treat CSF shunt infections and brain abscesses, too.

The myelotoxicity (especially the thrombocytopenia), is the most common serious adverse event caused by linezolid; ${ }^{59}$ it can be ameliorated or prevented by co-administration of pyridoxine (Vitamin B6). ${ }^{60-63}$ Both peripheral and optic neuropathy have been reported with prolonged use greater than four consecutive weeks. ${ }^{64,65}$ Lactic acidosis has also been reported and is not associated with duration of administration. ${ }^{65,66}$ Interaction exists between linezolid and serotonin-reuptake inhibitors (antidepressants drugs). In these last cases, a minority of patients might develop the so-called serotonin syndrome (fever, agitation with mental status changes and tremors). Due to its weak activity as a monoamine oxidase-inhibitor, linezolid should not be used concomitantly with agents, such as tramadol, pethidne, duloxetine, venlafaxine, milnacipran, sibutramine, chlorpeniramine, brompheniramine, cyproheptadine, citalopram, and paroxetine ${ }^{65-67}$ Drug metabolites may accumulate in the event of severe renal failure.

\section{Daptomycin}

Daptomycin is the first in a new class of antimicrobial agents: a lipopeptide antibiotic with activity against $S$. aureus (including methicillin-resistant strains), beta-hemolytic Groups A, B, C, and G Streptococci, and Enterococci, including ampicillin- and vancomycin-resistant strains. Both vancomycin-resistant Staphylococcus aureus and vancomycin-resistant Enterococci are susceptible to daptomycin. The mechanism of action of daptomycin is unique as the molecule causes a calcium ion dependent disruption of bacterial cell membrane potential resulting in an efflux of potassium, which inhibits RNA, DNA, and protein synthesis. Rare instances of resistance have occurred in clinical trials, although the mechanism of resistance has not yet been clearly identified to date. Daptomycin was shown to have a rapidly bactericidal effect in vitro against Gram-positive drug-resistant pathogens. Its activity is concentration-dependent and once daily dosing is associated with significant post-antibiotic effect.

Daptomycin is highly protein bound (around 92\%), with a terminal half-life of 8 hours, which allows for once daily dosing. Post-antibiotic effect proves to be dose dependent, and is reduced in the presence of albumin (i.e. exudates). The drug volume of distribution is low $(0.1 \mathrm{~L} / \mathrm{kg})$ and the Cmax $(54.6 \mathrm{mcg} / \mathrm{mL})$ is unchanged at steady state, and is achieved by day three of administration in humans. Cmax concentrations occur at the end of a 30-minutes infusion. Dosage needs to be reduced and dosing interval extended to every 48 hours in patients with reduced creatinine clearance $<30 \mathrm{~mL} / \mathrm{min}$; the same occurs for patients on either hemodialysis or peritoneal dialysis; the daptomycin dose is in these patients becomes $4 \mathrm{mg} / \mathrm{kg}$ every 48 hours. Daptomycin should be administered after hemodialysis as approximately $15 \%$ is cleared per 4 -hour hemodialysis session. On the other hand, no dose adjustments for hepatic dysfunction are required.

In early clinical trials conducted in the years 1980's1990 's, daptomycin was given in divided daily doses of $2 \mathrm{mg} /$ $\mathrm{kg}$ every 12 hours for skin and soft tissue infection and 3 $\mathrm{mg} / \mathrm{kg}$ every 12 hours for bacteremia, achieving good clini$\mathrm{cal}$ and bacteriological outcomes. However, rise in serum creatine phosphokinase (CPK), with myalgias, and muscle weakness led to initial abandonment of this promising antibiotic. However, myopathy was reversible upon drug cessation. With the advent of MRSA infections, daptomycin has been re-examined and resurrected, and its dosage has been increased to $4 \mathrm{mg} / \mathrm{kg}$ daily for skin and soft-tissue infection, ${ }^{68}$ and up to $6 \mathrm{mg} / \mathrm{kg}$ daily for bacteremia and endocarditis. ${ }^{69}$ Both indications have been approved by the United States FDA. Otherwise, daptomycin is not approved for the treatment of bacterial pneumonia; its efficacy is significantly compromised by its interaction with pulmonary surfactant. ${ }^{70}$ Significant drug-drug interaction occurs with the statins, and patients receiving HMG-CoA reductase inhibitors; these drugs should be suspended and avoided while the patient is undergoing a daptomycin course.

\section{Tigecycline}

Tigecycline is a novel glycylcycline molecule, which is a derivative of the tetracycline minocycline. Resistance to the tetracycline class is classically mediated by ribosomal protection mechanisms or by active efflux. Tigecycline has more potent activity against tetracycline-resistant organisms, and maintains a broad antibacterial spectrum against Gran-positive and also Gram-negative pathogens. Tigecycline binds more avidly to the ribosome and either does not induce efflux proteins or is not readily exported by efflux proteins. ${ }^{38}$ Resistant clinical isolates were associated with up-regulation of chromosomally mediated efflux pumps. Unlike original 
tetracyclines, tigecycline has a large volume of distribution (above $10 \mathrm{~L} / \mathrm{kg}$ ), the protein binding is approximately $68 \%$, the terminal half-life of elimination is 36 hours, and less than $15 \%$ of the native drug is excreted unchanged in the urine.

Clinical trials have been conducted in patients with complicated skin and soft tissue infections and intra-abdominal infections for which the drug gained its United States FDA approval.

Based on in vitro susceptibility data, tigecycline has a broad spectrum of activity against both Gram-positive cocci (including methicillin-resistant Staphylococci or MRSA, penicillin-resistant Streptococcus pneumoniae, betahemolytic group A and group B Streptococci, Enterococci (vancomycin-susceptible ones), and Listeria monocytogenes. Unlike other new agents for Gram-positive cocci, tigecycline also has extensive activity against Gram-negative pathogens, including Haemophilus influenzae, Neisseria spp, ${ }^{11}$ Enterobacteriaceae, and non-lactose fermenters other than Pseudomonas aeruginosa. The $\mathrm{MIC}_{90}$ values for Proteus spp., Providentia spp., and Burkholderia cepacia is $8 \mathrm{mcg} / \mathrm{mL}$, limiting its utility in infections caused by these afore mentioned pathogens.

Tigecycline needs no reduction in renal impairment and it is not dialyzable. Patients with severe hepatic dysfunction (Child-Pugh stage $\mathrm{C}$ liver disease) should receive a lower dosage. Tigecycline activity is dependent on the time above the MIC, and the drug concentrations should be above the MIC values for at least $50 \%$ of the dosing interval.

The expected adverse effects of tigeciclyne are primarily gastrointestinal in origin, with nausea, vomiting, diarrhea, and heartburn as the most frequent. As with all tetracyclines, tigecycline is contraindicated for pregnant females and for children less than 8 years of age. ${ }^{71}$ Drug interactions of tigecycline with either digoxin or warfarin do not alter the effect of either drug. Tigecycline does not inhibit metabolism mediated by the cytochrome $\mathrm{P} 450$ isoforms IA2, 2C8, 2C9, 2C19, 2D6 and 3A4, so that no drug-drug interaction is expected with drugs metabolized by these cytochrome isoforms.

\section{Dalbavancin}

Dalbavancin is a true second-generation lipoglycopeptide. Its unique pharmacokinetic profile allows once weekly dosing. It is not active against VRE, but has an excellent activity against MRSA, S. pyogenes and S. pneumoniae as well as vancomycin-susceptible Enterococci. It is bactericidal and synergistic with ampicillin against Van-A type Enterococci. The mechanism of action is the inhibition of the cell wall peptidoglycan cross-linking.

The daily dosage is $1000 \mathrm{mg}$ IV once, followed by $500 \mathrm{mg}$ IM 7 days later; the terminal half-life of dalbavancin is 9-12 days in humans due to protein binding greater than $95 \%$. Animal models of infection show excellent activity against
MRSA or GISA endocarditis, penicillin-resistant Streptococcus pneumoniae ${ }^{12}$ pneumonia or MRSA pouch infection, and septicemia due to Staphylococci, Streptococci or Enterococci. This antibiotic has been evaluated for catheterrelated bacteremia ${ }^{72}$ and skin and soft tissue infections. ${ }^{73}$ Dalbavancin was effective and well tolerated in adult patients with catheter-related bacteremia caused by coagulasenegative Staphylococci, MSSA and MRSA in a comparative trial with vancomycin. In skin and soft tissue infections, a 92-94\% microbiological and clinical response respectively was found in an open label phase 2 comparative dosing trial. ${ }^{73}$ Clinical success at follow-up visits for the two dose dalbavancin group was $80 \%$ for MRSA versus $50 \%$ for comparator therapy (which included beta-lactams, clindamycin, vancomycin and linezolid, respectively).

\section{Oritavancin}

Oritavancin is another derivative of vancomycin: it is a chloroeremomycin with the substitution of vancosamine by epivancosamine. It has a similar spectrum of activity to vancomycin but with consistently lower MIC values (<1 mg/L). No resistance to oritavancin has been observed among $S$. aureus strains including VISA strains, but VAN-A and VAN-B strains of Enterococci with reduced susceptibility to oritavancin have been obtained in vitro. The known mechanisms of resistance of oritavancin are: 1) complete elimination of D-Ala-entry precursors; 2) mutations in the VAN Sb sensor of VAN B cluster; or 3 ) the expression of Van $\mathrm{Z}$ (the precise function of which is still unknown).

Oritavancin shows rapid concentration bactericidaldependent activity with a concentration-dependent postantibiotic effect exerted against both VRE and MRSA. Oritavancin activity is negatively affected by large inoculum and its activity versus VRE was slightly reduced in stationary phase or in acidic foci of infection. In animal models, its efficacy has been demonstrated for experimental MRSA endocarditis and S. pneumoniae meningitis. ${ }^{74,75}$ In a reliable endocarditis model, the addition of gentamicin proved to be synergistic and able to prevent the emergence of resistant mutants. With regard to skin and soft tissue infections, oritavancin proved to be at least equivalent to vancomycin, for both clinical and bacteriological cure (about $78 \%$ cure rate). ${ }^{76}$

\section{Telavancin}

Telavancin is a rapidly bactericidal lipoglycopeptide analogue of vancomycin. The mechanism of action of this molecule is by inhibition of peptidoglycan chain formation through blockage of both the transpeptidation and transglycosylation steps; and by a direct effect on the bacterial membrane dissipating membrane potential and effecting changes in cellular permeability.

The in vitro activity of telavancin demonstrates enhanced activity against MRSA, penicillin-resistant $S$. pneumoniae, GISA and Van-A type Enterococci. Telavancin 
achieves a higher volume of distribution into tissues and a prolonged half-life. ${ }^{77} \mathrm{~A}$ high level of protein binding (93\%) occurs in human plasma and repetitive dosing does not lead to accumulation. The terminal half-life is $7-9$ hours at doses above $5 \mathrm{mg} / \mathrm{kg} .{ }^{78}$ Telavancin exhibit time-dependent killing activity. ${ }^{79}$

Telavancin and its comparators of vancomycin or betalactam agent have been compared in a phase 2 clinical trial for skin and skin-structure infections. Clinical cure rates were similar at $92 \%$ for telavancin versus $96 \%$ for comparator agents. Microbiologic rates of cure were noted to be 93\% in the telavancin group and $95 \%$ among the comparator group. ${ }^{80}$ For complicated skin and soft tissue infections, clinical cure rates were at $96 \%$ for telavancin and $90 \%$ for comparator agents. Microbiologic eradication was better with telavancin $(92 \%)$ versus comparator agents $(78 \%$, $\mathrm{p}=0.07){ }^{80}$ Telavancin is currently under assessment in phase 3 trials of hospital-acquired pneumonia.

Adverse events associated with telavancin among evaluated patients included vomiting, paresthesias, and dyspnea. Laboratory abnormalities included microalbuminemia and a decreased platelet count. ${ }^{81}$

\section{CLINICAL INDICATIONS OF NOVEL ANTIBI- OTICS WITH EXPANDED SPECTRUM AGAINST RESISTANT GRAM-POSITIVE COCCI}

\section{Skin and soft-tissue infections}

Skin and soft-tissue infections caused by Gram-positive cocci range from a simple cellulitis to life-threatening necrotizing fasciitis. All of the newer agents have been studied for such infections and have been found to be efficacious (Table 2). Most of the patients in these studies had less severe infections than necrotizing fasciitis as that infection requires a surgical approach as well as antibiotic therapy. All five FDAapproved agents, i.e. quinupristin/dalfopristin, linezolid, daptomycin, tigecycline, and vancomycin are appropriate choices for an effective treatment of Gram-positive pathogens. Only tigecycline has activity against Gram-negative bacilli pathogens. So, tigecycline may have a major role for diabetic foot infections and infected decubitus ulcers which may be co-infected by anaerobic bacteria and aerobic Gramnegative bacilli, in addition to Gram-positive cocci.

\section{Bone and joint infections}

With regard to osteomyelitis and joint infections, Grampositive cocci largely predominate over other microbial pathogens. S. aureus, and both MSSA and MRSA, as well as coagulase-negative Staphylococci account for over 50\% of recovered pathogens. Unfortunately, only few studies have prospectively investigated the above-mentioned newer antibiotics in these infections. ${ }^{56,57}$ Aneziokoro et al. evaluated 20 patients who received linezolid for osteomyelitis for 6 weeks or more in a retrospective non-comparative study ${ }^{82}$ Fifty-five percent of cases (11 patients) achieved a cure with follow-up periods ranging from 6 to 49 months (median of 36 months). Prospective comparative studies of efficacy in bone and joint infections have not been reported to date. In two retrospective studies, 22 patients with osteomyelitis and three subjects with septic joint infections were treated with daptomycin. ${ }^{83,84}$ MRSA was the predominant pathogen in over $75 \%$ of patients. Daptomycin was used as salvage therapy, and its usual dose was 6 $\mathrm{mg} / \mathrm{kg} /$ day. Clinical success rate was about $90 \%$; follow up periods were one year or less. Limited data has been published with respect to bone and joint infections for dalbavancin, tigecycline or quinupristin/dalfopristin in humans. In a rabbit model of MRSA osteomyelitis, the combination of rifampin and tigecycline was compared to vancomycin with or without rifampicin, tigecycline alone, and vancomycin alone. ${ }^{85}$ All regimens were effective (in about $90 \%$ of episodes). Untreated rabbits had spontaneous cure in $26 \%$ of cases (4/15). Tigecycline concentrations are higher in infected bone than in non-infected bone. A rabbit model of quinupristin/dalfopristin prosthetic joint infection with MRSA was compared to vancomycin with or without rifampicin, showing an equivalent outcome. ${ }^{86}$

\section{Pneumonia and lower respiratory tract infections}

Pneumonia due to Gram-positive cocci is common. In the community, infection is usually due to $S$. pneumoniae and occasionally S. aureus. Hospital-acquired pneumonia (HAP) is often caused by MRSA organisms. Linezolid was comparable to vancomycin in the therapy of MRSA-associated VAP, although a trend was seen for linezolid superiority. ${ }^{53,54}$ Daptomycin is not indicated for pneumonia due to its interaction with surfactant, ${ }^{70}$ while tigecycline is undergoing clinical evaluation. Quinupristin/dalfopristin has been compared to vancomycin for hospital-acquired pneumonia. ${ }^{47}$ One hundred and seventy one patients had similar clinical response rates of about $57 \%$ respectively. Drug discontinuation adverse events occurred more frequently in the quinupristin/dalfopristin group (15\%), as compared to vancomycin. Only two isolated of the 87 overall strains were shown to have decreased susceptibility to quinupristin/dalfopristin during and after treatment.

\section{Intra-abdominal infections}

Of the newer antibiotics, only tigecycline has been approved for intra-abdominal infections. As mentioned, tigecycline's broader spectrum of activity includes Gram-negative bacilli and anaerobic bacilli. Linezolid, daptomycin, and quinupristin/dalfopristin can be used in combination with antibiotics with Gram-negative spectrum of activity such as aztreonam, and especially carbapenems, fluoroquinolones, and aminoglycosides. Of concern, quinupristin/dalfopristin has no activity against $\mathrm{E}$. faecalis. 


\section{Bacteremia and endocarditis}

Daptomycin and quinipristin/dalfopristin have been approved by the United States FDA organisms for the treatment of Gram-positive bacteremia. In addition, daptomycin has been approved for use in S. aureus right-sided endocarditis ${ }^{87}$ Dalbavancin, linezolid, tigecycline and oritavancin have not yet been approved for bacteremia due to Grampositive cocci. Linezolid has been evaluated for Gram-positive bacteria. ${ }^{55,88,89}$ Among 108 bacteremic patients receiving linezolid, eradication was seen in $91 \%$ and clinical cure was seen in $94 \%$ of the episodes. ${ }^{55}$ On the other hand, linezolid is still not approved for catheter-related becteremia and endocarditis. A randomized study of 726 patients with catheter-related bacteremia received linezolid or vancomycin; an excess number of deaths were seen for patients receiving linezolid due mainly to Gram-negative rods implicated in these infections. ${ }^{90}$ Based on 23 case reports and three case series, a total of $63 \%(21 / 33)$ of patients with endocarditis were successfully cured after linezolid administration. ${ }^{91}$ MRSA and vancomycin-intermediate $S$. aureus were the most commonly isolated cocci $24.2 \%$ and $30.3 \%$ of cases, respectively). Five cases were successfully treated with linezolid monotherapy.

\section{POTENTIAL SYNERGISTIC INTERACTIONS OF NEWER ANTIBIOTICS: IN VITRO STUDIES}

In vitro interaction between the new anti-staphylococcal antibiotics were virtually always indifferent, therefore leading to a possible additive effect, although a few experiments showed possible (Table 4). . $9,92-95,98-107$ For instance, a synergistic interaction was found for quinupristin/dalfopristin and vancomycin in two independent studies. ${ }^{92,93}$

Table 4. Some experimental studies conducted in vitro or on animal models, regarding possible interactions between the different antimicrobial agents effective on Gram-positive Cocci89,92-95,98-107

\begin{tabular}{|l|l|l|l}
\hline $\begin{array}{l}\text { Reference } \\
\text { quotation }\end{array}$ & Combination & Pathogens & Interaction \\
\hline 97 & Daptomycin + vancomycin & GISA & Additive \\
\hline 97 & Daptomycin + gentamicin & GISA & Additive \\
\hline 93 & Daptomycin + gentamicin & MSSA/MRSA & Enhanced time-kill \\
\hline 98 & Daptomycin + gentamicin & MSSA/MRSA & Increased killing \\
\hline 99 & Daptomycin + rifampicin & MRSA & Additive \\
\hline 100 & Daptomycin + gentamicin + rifampicin & MRSA & Additive \\
\hline 97 & Linezolid + vancomycin & GISA & Additive \\
\hline 94 & Linezolid + vancomycin & MSSA/MRSA & Antagonistic \\
\hline 89,95 & Linezolid + vancomycin & MRSA & Indifferent \\
\hline 101 & Linezolid + vancomycin & MSSA/MRSA/MRSE & Increased killing \\
\hline 94,98 & Linezolid + gentamicin & MSSA/MRSA & Indifferent \\
\hline 95 & Linezolid + gentamicin & MSSA/MRSA & Antagonistic \\
\hline 94 & Linezolid + rifampicin & MSSA/MRSA & Synergistic \\
\hline 95 & Linezolid + rifampicin & MSSA & Indifferent \\
\hline 102 & Linezolid + rifampicin & MRSA & Increased killing \\
\hline 101 & Linezolid + quinupristin/dalfopristin & GISA & Additive-Synergistic \\
\hline 97 & Quinupristin/dalfopristin + vancomycin & MSSA/MRSA & Additive \\
\hline 103 & Quinupristin/dalfopristin + vancomycin & MRSA & Increased killing \\
\hline 101 & Quinupristin/dalfopristin + vancomycin & MSSA/MRSA & Synergistic \\
\hline 92 & Quinupristin/dalfopristin + vancomycin & GISA & Indifferent \\
\hline 97 & Quinupristin/dalfopristin + gentamicin & MSSA & Increased killing \\
\hline 104 & Quinupristin/dalfopristin + rifampicin & MRSA & Synergistic \\
\hline 105 & Quinupristin/dalfopristin + rifampicin & MRSA/GISA & Increased killing \\
\hline 106 & Tigecycline + vancomycin & Indifferent \\
\hline 107 & Tigecycline + rifampicin & & \\
\hline 106 & & & MRSA \\
\hline
\end{tabular}


On the other hand, antagonistic interactions were demonstrated for the combination of linezolid plus vancomycin, ${ }^{94}$ and linezolid plus gentamicin. ${ }^{95}$ It should be emphasized that in vitro interaction may not translate into clinical efficacy. Quinupristin/daflopristin in combination with vancomycin appeared to be favourable for the management of MRSA infections responding poorly to vancomycin. ${ }^{96}$ However, we have to specify that the MRSA isolates were of a specific genotype, accessory gene regulator (agr), which has been linked to vancomycin treatment failure. ${ }^{96}$ Nevertheless, such information may be useful if innovative combination therapy needs to be administered to severely ill patients with invasive $S$. aureus infection unresponsive to monotherapy.

Controlled clinical trials using combinations including these new agents are indicated for patients with severe, lifethreatening infections caused by gram-positive cocci, and randomized trials are strongly warranted in this somewhat unexplored field.

\section{REFERENCES}

1. Foster JK, Lentino JR, Strodtman R, DiVincenzo C. Comparison of in vitro activity of quinolone antibiotics and vancomycin against gentamicin- and methicillin-resistant Staphylococcus aureus by time-kill kinetic studies. Antimicrob Agents Chemother 1986;30:823-7.

2. Tallent SM, Bischoff T, Climo M, Ostrowsky B, Wenzel RP, Edmond MB. Vancomycin susceptibility of oxacillin-resistant Staphylococcus aureus isolates causing nosocomial bloodstream infections. J Clin Microbiol 2002; 40:2249-50.

3. Sieradzki K, Leski T, Dick J, Borio L, Tomasz A. Evolution of a vancomycin-intermediate Staphylococcus aureus strain in vivo: multiple changes in the antibiotic resistance phenotypes of a single lineage of methicillin-resistant $S$. aureus under the impact of antibiotics administered for chemotherapy. J Clin Microbiol 2003; 41:1687-93.

4. Murray BE. Vancomycin-resistant enterococci. Am J Med 1997; 102:284-93.

5. Okuma K, Iwakawa K, Turnidge JD et al. Dissemination of new methicillin-resistant Staphylococcus aureus clones in the community. J Clin Microbiol 2002; 40:4289-94.

6. Schwalbe RS, Stapleton JT, Gilligan PH. Emergence of vancomycin resistance in coagulase-negative staphylococci. $\mathrm{N}$ Engl J Med 1987; 316:927-31.

7. Hiramatsu K, Hanaki H, Ino T, Yabuta K, Oguri T, Tenover FC. Methicillin-resistant Staphylococcus aureus clinical strain with reduced vancomycin susceptibility. J Antimicrob Chemother 1997; 40:135-6.

8. Appelbaum PC. MRSA-the tip of the iceberg. Clin Microbiol Infect 2006; 12 Suppl 2:3-10.

9. MMWR. Staphylococcus aureus resistant to vancomycin-United States 2002. Vol. 51 (26): 565.567RE, 2002.

10. Chang S, Sievert DM, Hageman JC et al. Infection with vancomycin-resistant Staphylococcus aureus containing the vanA resistance gene. N Engl J Med 2003; 348:1342-7.

11. King MD, Humphrey BJ, Wang YF, Kourbatova EV, Ray SM, Blumberg HM. Emergence of community-acquired methicillin-resistant Staphylococcus aureus USA 300 clone as the predominant cause of skin and soft-tissue infections. Ann Intern Med 2006;144:309-17.

12. Moellering RC, Jr. The growing menace of community-acquired methicillin-resistant Staphylococcus aureus. Ann Intern Med 2006; 144:368-70.
13. Noskin GA, Rubin RJ, Schentag JJ et al. The burden of Staphylococcus aureus infections on hospitals in the United States: an analysis of the 2000 and 2001 Nationwide Inpatient Sample Database. Arch Intern Med 2005; 165:1756-61.

14. Rupp ME, Archer GL. Coagulase-negative Staphylococci: pathogens associated with medical progress. Clin Infect Dis 1994; 19:231-43; quiz 244-5.

15. Schaaff F, Reipert A, Bierbaum G. An elevated mutation frequency favors development of vancomycin resistance in Staphylococcus aureus. Antimicrob Agents Chemother 2002; 46:3540-8.

16. Chang FY, Peacock JE, Jr, Musher DM et al. Staphylococcus aureus bacteremia: recurrence and the impact of antibiotic treatment in a prospective multicenter study. Medicine (Baltimore) 2003; 82:333-9.

17. Markowitz N, Quinn EL, Saravolatz LD. Trimethoprim-sulfamethoxazole compared with vancomycin for the treatment of Staphylococcus aureus infection. Ann Intern Med 1992; 117:390-8.

18. Becker K, Friedrich AW, Lubritz G, Weilert M, Peters G, Von Eiff C. Prevalence of genes encoding pyrogenic toxin superantigens and exfoliative toxins among strains of Staphylococcus aureus isolated from blood and nasal specimens. J Clin Microbiol 2003;41:1434-9.

19. Lowy FD. Staphylococcus aureus infections. N Engl J Med 1998; 339:520-32.

20. Sakoulas G, Moellering RC, Jr, Eliopoulos GM. Adaptation of methicillin-resistant Staphylococcus aureus in the face of vancomycin therapy. Clin Infect Dis 2006; 42 Suppl 1:S40-50.

21. Donlan RM, Costerton JW. Biofilms: survival mechanisms of clinically relevant microorganisms. Clin Microbiol Rev 2002; 15:167-93.

22. Caiazza NC, O’Toole GA. Alpha-toxin is required for biofilm formation by Staphylococcus aureus. J Bacteriol 2003; 185:3214-7.

23. von Eiff C, Peters G, Heilmann C. Pathogenesis of infections due to coagulase-negative Staphylococci. Lancet Infect Dis 2002; 2:677-85

24. Yu VL, Chiou CC, Feldman C et al. An international prospective study of pneumococcal bacteremia: correlation with in vitro resistance, antibiotics administered, and clinical outcome. Clin Infect Dis 2003; 37:230-7.

25. Peterson LR. Penicillins for treatment of pneumococcal pneumonia: does in vitro resistance really matter? Clin Infect Dis 2006; 42:224-33.

26. Lonks JR, Garau J, Gomez L et al. Failure of macrolide antibiotic treatment in patients with bacteremia due to erythromycin-resistant Streptococcus pneumoniae. Clin Infect Dis 2002; 35:556-64

27. Schentag JJ, Klugman KP, Yu VL et al. Streptococcus pneumoniae bacteremias: pharmacodynamic correlations with outcome and macrolide resistance: a controlled study. Int J Antimicrob Agents 2007; 30:264-9.

28. Greenberg D DR, Klugman K, Madhi SA et al. Streptococcus pneumoniae serotypes causing meningitis in children and adults. Proceedings of the 14th ICAAC Conference. Washington, DC., 2004.

29. Carpenter CF, Chambers HF. Daptomycin: another novel agent for treating infections due to drug-resistant gram-positive pathogens. Clin Infect Dis 2004; 38:994-1000.

30. Fenton C, Keating GM, Curran MP. Daptomycin. Drugs 2004; 64:445-55.

31. Steenbergen JN, Alder J, Thorne GM, Tally FP. Daptomycin: a lipopeptide antibiotic for the treatment of serious Grampositive infections. J Antimicrob Chemother 2005; 55:283-8. 
32. Schriever CA, Fernandez C, Rodvold KA, Danziger LH. Daptomycin: a novel cyclic lipopeptide antimicrobial. Am J Health Syst Pharm 2005; 62:1145-58.

33. Moellering RC. Linezolid: the first oxazolidinone antimicrobial. Ann Intern Med 2003; 138:135-42.

34. Birmingham MC, Rayner CR, Meagher AK, Flavin SM, Batts $\mathrm{DH}$, Schentag JJ. Linezolid for the treatment of multidrugresistant, gram-positive infections: experience from a compassionate-use program. Clin Infect Dis 2003; 36:159-68.

35. LaPlante KL, Rybak MJ. Daptomycin - a novel antibiotic against Gram-positive pathogens. Expert Opin Pharmacother 2004; 5:2321-31.

36. Jeu L, Fung HB. Daptomycin: a cyclic lipopeptide antimicrobial agent. Clin Ther 2004; 26:1728-57.

37. Alder JD. Daptomycin: a new drug class for the treatment of Gram-positive infections. Drugs Today (Barc) 2005; 41:81-90.

38. Livermore DM. Tigecycline: what is it, and where should it be used? J Antimicrob Chemother 2005; 56:611-4.

39. Pankey GA. Tigecycline. J Antimicrob Chemother 2005; 56:470-80.

40. Van Bambeke F, Van Laethem Y, Courvalin P, Tulkens PM. Glycopeptide antibiotics: from conventional molecules to new derivatives. Drugs 2004; 64:913-36.

41. Virginlar N. MA. Glycopeptides (Dalbavancin, Oritavancin, Teicoplanin, Vancomycin). In: Yu VL, Eds. Antimicrobial Therapy and Vaccines. Vol. II: Antimicrobial Agents: www.antimicrobe.org, 2004.

42. Hershberger E, Donabedian S, Konstantinou K, Zervos MJ. Quinupristin-dalfopristin resistance in gram-positive bacteria: mechanism of resistance and epidemiology. Clin Infect Dis 2004; 38:92-8.

43. Speciale A, La Ferla K, Caccamo F, Nicoletti G. Antimicrobial activity of quinupristin/dalfopristin, a new injectable streptogramin with a wide Gram-positive spectrum. Int J Antimicrob Agents 1999; 13:21-8.

44. Moellering RC, Linden PK, Reinhardt J, Blumberg EA, Bompart F, Talbot GH. The efficacy and safety of quinupristin/dalfopristin for the treatment of infections caused by vancomycin-resistant Enterococcus faecium. Synercid Emergency-Use Study Group. J Antimicrob Chemother 1999; 44:251-61.

45. Nichols RL, Graham DR, Barriere SL et al. Treatment of hospitalized patients with complicated gram-positive skin and skin structure infections: two randomized, multicentre studies of quinupristin/dalfopristin versus cefazolin, oxacillin or vancomycin. Synercid Skin and Skin Structure Infection Group. J Antimicrob Chemother 1999; 44:263-73.

46. Meka VG, Pillai SK, Sakoulas G et al. Linezolid resistance in sequential Staphylococcus aureus isolates associated with a T2500A mutation in the 23S rRNA gene and loss of a single copy of rRNA. J Infect Dis 2004; 190:311-7.

47. Fagon J, Patrick H, Haas DW et al. Treatment of grampositive nosocomial pneumonia. Prospective randomized comparison of quinupristin/dalfopristin versus vancomycin. Nosocomial Pneumonia Group. Am J Respir Crit Care Med 2000; 161:753-62.

48. Drew RH, Perfect JR, Srinath L, Kurkimilis E, Dowzicky M, Talbot GH. Treatment of methicillin-resistant Staphylococcus aureus infections with quinupristin-dalfopristin in patients intolerant of or failing prior therapy. For the Synercid Emergency-Use Study Group. J Antimicrob Chemother 2000; 46:775-84.
49. Carver PL, Whang E, VandenBussche HL, Kauffman CA, Malani PN. Risk factors for arthralgias or myalgias associated with quinupristin-dalfopristin therapy. Pharmacotherapy 2003; 23:159-64.

50. Raad I, Hachem R, Hanna H. Relationship between myalgias/ arthralgias occurring in patients receiving quinupristin/dalfopristin and biliary dysfunction. J Antimicrob Chemother 2004; 53:1105-8.

51. Stalker DJ, Jungbluth GL. Clinical pharmacokinetics of linezolid, a novel oxazolidinone antibacterial. Clin Pharmacokinet 2003; 42:1129-40.

52. Weigelt J, Itani K, Stevens D, Lau W, Dryden M, Knirsch C. Linezolid versus vancomycin in treatment of complicated skin and soft tissue infections. Antimicrob Agents Chemother 2005; 49:2260-6.

53. Rubinstein E, Cammarata S, Oliphant T, Wunderink R. Linezolid (PNU-100766) versus vancomycin in the treatment of hospitalized patients with nosocomial pneumonia: a randomized, double-blind, multicenter study. Clin Infect Dis 2001; 32:402-12.

54. Wunderink RG, Rello J, Cammarata SK, Croos-Dabrera RV, Kollef MH. Linezolid vs vancomycin: analysis of two doubleblind studies of patients with methicillin-resistant Staphylococcus aureus nosocomial pneumonia. Chest 2003; 124:1789-97.

55. Rayner CR, Forrest A, Meagher AK, Birmingham MC, Schentag JJ. Clinical pharmacodynamics of linezolid in seriously ill patients treated in a compassionate use programme. Clin Pharmacokinet 2003; 42:1411-23.

56. Rayner CR, Baddour LM, Birmingham MC, Norden C, Meagher AK, Schentag JJ. Linezolid in the treatment of osteomyelitis: results of compassionate use experience. Infection 2004; 32:814.

57. Razonable RR, Osmon DR, Steckelberg JM. Linezolid therapy for orthopedic infections. Mayo Clin Proc 2004; 79:1137-44.

58. Cook AM, Ramsey CN, Martin CA, Pittman T. Linezolid for the treatment of a heteroresistant Staphylococcus aureus shunt infection. Pediatr Neurosurg 2005; 41:102-4. 59. Rho JP, Sia IG, Crum BA, Dekutoski MB, Trousdale RT. Linezolid-associated peripheral neuropathy. Mayo Clin Proc 2004; 79:927-30.

60. Spellberg B, Yoo T, Bayer AS. Reversal of linezolid-associated cytopenias, but not peripheral neuropathy, by administration of vitamin B6. J Antimicrob Chemother 2004; 54:832-5.

61. Young LS. Hematologic effects of linezolid versus vancomycin. Clin Infect Dis 2004; 38:1065-6.

62. Rao N, Ziran BH, Wagener MM, Santa ER, Yu VL. Similar hematologic effects of longterm linezolid and vancomycin therapy in a prospective observational study of patients with orthopedic infections. Clin Infect Dis 2004; 38:1058-64.

63. Nasraway SA, Shorr AF, Kuter DJ, O'Grady N, Le VH, Cammarata SK. Linezolid does not increase the risk of thrombocytopenia in patients with nosocomial pneumonia: comparative analysis of linezolid and vancomycin use. Clin Infect Dis 2003; 37:1609-16.

64. Kulkarni K, Del Priore LV. Linezolid induced toxic optic neuropathy. Br J Ophthalmol 2005; 89:1664-5.

65. Narita MT, B Yu VL. Linezolid-associated peripheral and optic neuropathy, lactic acidosid and serotonin syndrome: a review. Pharmacotherapy 2007; 27:1189-97.

66. Soriano A, Miro O, Mensa J. Mitochondrial toxicity associated with linezolid. N Engl J Med 2005; 353:2305-6.

67. Bernard L, Stern R, Lew D, Hoffmeyer P. Serotonin syndrome after concomitant treatment with linezolid and citalopram. Clin Infect Dis 2003; 36:1197. 
68. Arbeit RD, Maki D, Tally FP, Campanaro E, Eisenstein BI. The safety and efficacy of daptomycin for the treatment of complicated skin and skin-structure infections. Clin Infect Dis 2004; 38:1673-81.

69. Fowler VG, Cosgrove S. Abrutyn E, et al. Daptomycin vs. Standard Therapy for Staphylococcus aureus Bacteremia (SAB) and Infective Endocarditis (SAIE). 45th Annual Interscience Congress on Antimicrobial Agents and Chemotherapy. Washington DC, 2005.

70. LaPlante KL, Rybak, M.J. Daptomycin. Antimicrobial Therapy and Vaccines. Vol. II: Antimicrobial Agents: www.antimicrobe.org.

71. Zhanel GG, Homenuik K, Nichol K et al. The glycylcyclines: a comparative review with the tetracyclines. Drugs 2004; 64:63-88.

72. Raad I, Darouiche R, Vazquez J et al. Efficacy and safety of weekly dalbavancin therapy for catheter-related bloodstream infection caused by gram-positive pathogens. Clin Infect Dis 2005; 40:374-80.

73. Seltzer E, Dorr MB, Goldstein BP, Perry M, Dowell JA, Henkel T. Once-weekly dalbavancin versus standard-of-care antimicrobial regimens for treatment of skin and soft-tissue infections. Clin Infect Dis 2003; 37:1298-303.

74. Kaatz GW, Seo SM, Aeschlimann JR, Houlihan HH, Mercier RC, Rybak MJ. Efficacy of LY333328 against experimental methicillin-resistant Staphylococcus aureus endocarditis. Antimicrob Agents Chemother 1998; 42:981-3.

75. Gerber J, Smirnov A, Wellmer A et al. Activity of LY333328 in experimental meningitis caused by a Streptococcus pneumoniae strain susceptible to penicillin. Antimicrob Agents Chemother 2001; 45:2169-72.

76. Giamarellou H ORW, Harris H, Owen S, Porter S, Loutit J. Phase 3 trial comparing 3-7 days of oritavancin vs. 10-14 days of vancomycin/cephalexin in the treatment of patients with complicated skin and skin structure infections (CSSI). In: Program and abstracts of the 43rd ICAAC Conference, Chicago, IL, September 14-17,2003, 2003. American Society of Microbiology.

77. Barrett JF. Recent developments in glycopeptide antibacterials. Curr Opin Investig Drugs 2005; 6:781-90.

78. Shaw JP, Seroogy J, Kaniga K, Higgins DL, Kitt M, Barriere S. Pharmacokinetics, serum inhibitory and bactericidal activity, and safety of telavancin in healthy subjects. Antimicrob Agents Chemother 2005; 49:195-201.

79. Hegde SS, Reyes N, Wiens $\mathrm{T}$ et al. Pharmacodynamics of telavancin (TD-6424), a novel bactericidal agent, against gram-positive bacteria. Antimicrob Agents Chemother 2004; 48:3043-50.

80. Stryjewski ME, Chu VH, O'Riordan WD et al. Telavancin versus standard therapy for treatment of complicated skin and skin structure infections caused by gram-positive bacteria: FAST 2 study. Antimicrob Agents Chemother 2006; 50:862-7.

81. Stryjewski ME, O'Riordan WD, Lau WK et al. Telavancin versus standard therapy for treatment of complicated skin and soft-tissue infections due to gram-positive bacteria. Clin Infect Dis 2005; 40:1601-7.

82. Aneziokoro CO, Cannon JP, Pachucki CT, Lentino JR. The effectiveness and safety of oral linezolid for the primary and secondary treatment of osteomyelitis. J Chemother 2005; 17:643-50.

83. Finney MS, Crank CW, Segreti J. Use of daptomycin to treat drug-resistant Gram-positive bone and joint infections. Curr Med Res Opin 2005; 21:1923-6.
84. Anthony S.J. HM, Angelos E, Stratton CW. Clinical Experience with daptomycin in Patients with Orthopedic-Related Infections. 43rd IDSA Annual Meeting 2005. San Francisco, CA, 2005.

85. Yin LY, Lazzarini L, Li F, Stevens CM, Calhoun JH. Comparative evaluation of tigecycline and vancomycin, with and without rifampicin, in the treatment of methicillin-resistant Staphylococcus aureus experimental osteomyelitis in a rabbit model. J Antimicrob Chemother 2005; 55:995-1002.

86. Howden BP, Ward PB, Charles PG et al. Treatment outcomes for serious infections caused by methicillin-resistant Staphylococcus aureus with reduced vancomycin susceptibility. Clin Infect Dis 2004; 38:521-8.

87. Fowler VG, Jr., Boucher HW, Corey GR et al. Daptomycin versus standard therapy for bacteremia and endocarditis caused by Staphylococcus aureus. N Engl J Med 2006; 355:653-65.

88. Woods CW, Cheng AC, Fowler VG, Jr et al. Endocarditis caused by Staphylococcus aureus with reduced susceptibility to vancomycin. Clin Infect Dis 2004; 38:1188-91.

89. Chiang FY, Climo M. Efficacy of linezolid alone or in combination with vancomycin for treatment of experimental endocarditis due to methicillin-resistant Staphylococcus aureus. Antimicrob Agents Chemother 2003; 47:3002-4.

90. FDA. Information for Healthcare Professionals: Linezolid (marketed as Zyvox) March 16, 2007, 2007.

91. Falagas ME, Manta KG, Ntziora F, Vardakas KZ. Linezolid for the treatment of patients with endocarditis: a systematic review of the published evidence. J Antimicrob Chemother 2006; 58:273-80.

92. Kang SL, Rybak MJ. In-vitro bactericidal activity of quinupristin/dalfopristin alone and in combination against resistant strains of Enterococcus species and Staphylococcus aureus. J Antimicrob Chemother 1997; 39 Suppl A:33-9.

93. Tsuji BT, Rybak MJ. Short-course gentamicin in combination with daptomycin or vancomycin against Staphylococcus aureus in an in vitro pharmacodynamic model with simulated endocardial vegetations. Antimicrob Agents Chemother 2005; 49:2735-45.

94. Grohs P, Kitzis MD, Gutmann L. In vitro bactericidal activities of linezolid in combination with vancomycin, gentamicin, ciprofloxacin, fusidic acid, and rifampin against Staphylococcus aureus. Antimicrob Agents Chemother 2003; 47:418-20.

95. Jacqueline C, Caillon J, Le Mabecque V et al. In vitro activity of linezolid alone and in combination with gentamicin, vancomycin or rifampicin against methicillin-resistant Staphylococcus aureus by time-kill curve methods. J Antimicrob Chemother 2003; 51:857-64.

96. Moise-Broder PA, Sakoulas G, Eliopoulos GM, Schentag JJ, Forrest A, Moellering RC, Jr Accessory gene regulator group II polymorphism in methicillin-resistant Staphylococcus aureus is predictive of failure of vancomycin therapy. Clin Infect Dis 2004; 38:1700-5.

97. Tsuji BT, Rybak MJ. Etest synergy testing of clinical isolates of Staphylococcus aureus demonstrating heterogeneous resistance to vancomycin. Diagn Microbiol Infect Dis 2006; 54:73-7.

98. LaPlante KL, Rybak MJ. Impact of high-inoculum Staphylococcus aureus on the activities of nafcillin, vancomycin, linezolid, and daptomycin, alone and in combination with gentamicin, in an in vitro pharmacodynamic model. Antimicrob Agents Chemother 2004; 48:4665-72.

99. Sakoulas G, Eliopoulos GM, Alder J, Eliopoulos CT. Efficacy of daptomycin in experimental endocarditis due to methicillinresistant Staphylococcus aureus. Antimicrob Agents Chemother 2003; 47:1714-8. 
100. Baltch A RW, Bopp L. et al. Killing of methicillin-resistant Staphylococcus aureus by daptomycin, gentamicin, and rifampin, singly and in combination, in broth and in human monocytederived macrophages, with and without GM-CSF and Interferon Activation. Proceedings of the 15th ICAAC Conference, 2005, Abstract E-1741, American Society of Microbiology, Washington, 2005.

101. Allen GP, Cha R, Rybak MJ. In vitro activities of quinupristin-dalfopristin and cefepime, alone and in combination with various antimicrobials, against multidrug-resistant Staphylococci and Enterococci in an in vitro pharmacodynamic model. Antimicrob Agents Chemother 2002; 46:2606-12.

102. Dailey CF, Pagano PJ, Buchanan LV, Paquette JA, Haas JV, Gibson JK. Efficacy of linezolid plus rifampin in an experimental model of methicillin-susceptible Staphylococcus aureus endocarditis. Antimicrob Agents Chemother 2003; 47:2655-8.

103. Kang SL, Rybak MJ, McGrath BJ, Kaatz GW, Seo SM. Pharmacodynamics of levofloxacin, ofloxacin, and ciprofloxacin, alone and in combination with rifampin, against methicillin-susceptible and -resistant Staphylococcus aureus in an in vitro infection model. Antimicrob Agents Chemother 1994; 38:2702-9.
104.Zarrouk V, Bozdogan B, Leclercq R et al. Activities of the combination of quinupristin-dalfopristin with rifampin in vitro and in experimental endocarditis due to Staphylococcus aureus strains with various phenotypes of resistance to macrolidelincosamide-streptogramin antibiotics. Antimicrob Agents Chemother 2001; 45:1244-8.

105. Sambatakou H, Giamarellos-Bourboulis EJ, Grecka P, Chryssouli Z, Giamarellou H. In vitro activity and killing effect of quinupristin/dalfopristin (RP59500) on nosocomial Staphylococcus aureus and interactions with rifampicin and ciprofloxacin against methicillin-resistant isolates. J Antimicrob Chemother 1998; 41:349-55.

106. Petersen PJ, Labthavikul P, Jones $\mathrm{CH}$, Bradford PA. In vitro antibacterial activities of tigecycline in combination with other antimicrobial agents determined by chequerboard and timekill kinetic analysis. J Antimicrob Chemother 2006; 57:573-6.

107. Mercier RC, Kennedy C, Meadows C. Antimicrobial activity of tigecycline (GAR-936) against Enterococcus faecium and Staphylococcus aureus used alone and in combination. Pharmacotherapy $2002 ; 22: 1517-23$. 